\title{
Genetic Corner: Diaphragmatic Hernia in an Infant with a Type II Distal Deletion of 22q11.2 (LCR22E-F)
}

Robin Dawn Clark, MD

\section{Case summary:}

A 34-day old female with a left-sided congenital diaphragmatic hernia $(\mathrm{CDH})$ was born at 33w 3d gestation to an obese 18-year old G1P0 mother by vaginal delivery. The mother was admitted for preterm labor at $32 \mathrm{w}$. During that admission, polyhydramnios, mild ventriculomegaly, $\mathrm{CDH}$, and pre-eclampsia were diagnosed. Amniotic reduction was performed four times. The baby was delivered nine days after the mother was admitted. The mother denied teratogenic exposures, diabetes, or trauma. Her BMI was 58.82 $\mathrm{kg} / \mathrm{m}^{2}$. She had mild spotting for two days at $26 \mathrm{w}$. Apgar scores were 6 and 7 . BW was $2.225 \mathrm{~g}$ (Z-score 0.58$), \mathrm{BL} 43 \mathrm{~cm}$ (Z-score $-0.08), \mathrm{HC} 31 \mathrm{~cm}$ (Z-score 0.58). Her diaphragmatic hernia was repaired with mesh on day 18 . An echocardiogram after surgery showed a moderate to large patent ductus arteriosus, $L>R$, patent foramen ovale, mild dilation of the left ventricle, and mild elevation of pulmonary pressures. She is currently tolerating the slow advancement of feedings.

"Minor dysmorphic features were appreciated on the physical exam but did not suggest a particular syndrome. The forehead was square and prominent. The ear lobes were uplifted. The philtrum was long, and the upper lip was thin. The nose was broad with upturned nasal tip and anteverted nares. There was clinodactyly of the little fingers."

Minor dysmorphic features were appreciated on the physical exam but did not suggest a particular syndrome. The forehead was square and prominent. The ear lobes were uplifted. The philtrum was long, and the upper lip was thin. The nose was broad with upturned nasal tip and anteverted nares. There was clinodactyly of the little fingers. The baby responded to light touch and was jittery during the exam.

\section{Family history:}

The mother is 18 years old, and the father is 19. The parents are from Mexico and deny consanguinity. The family history was negative for other relatives with diaphragmatic hernia or other congenital anomalies.

\section{Laboratory tests:}

An amniocentesis had been performed nine days prior to delivery, but the normal prenatal microarray results, $\operatorname{arr}(1-22, \mathrm{X}) \times 2$, did not return until after delivery. By that time, a postnatal microarray had been sent to the same laboratory. That study, interpreted with postnatal reporting criteria, revealed a $655 \mathrm{~kb}$ deletion from 22q11.22 to 22q11.23, spanning low copy repeat regions LCR22$\mathrm{E}$ and LCR22-F (distal type II deletion). The deleted interval involved eight known genes (MIR650, MIR5571, IGLL5, RSPH14, GNAZ, RAB36, BCR, FBXW4P1), none of which are known to be associated with diaphragmatic hernia.

Parental follow-up testing for this variant is in progress.

"That study, interpreted with postnatal reporting criteria, revealed a $655 \mathrm{~kb}$ deletion from 22q11.22 to 22q11.23, spanning low copy repeat regions LCR22-E and LCR22-F (distal type II deletion). The deleted interval involved eight known genes (MIR650, MIR5571, IGLL5, RSPH14, GNAZ, RAB36, BCR, FBXW4P1), none of which are known to be associated with diaphragmatic hernia."

\section{Discussion:}

In reviewing this case, I was struck by these three points:

1. A pathogenic deletion was detected on the postnatal microarray, but the prenatal microarray had been normal

2. This child has an atypical, distal, smaller 22q11.2 deletion that is distinct from the more common deletion associated with DiGeorge syndrome

3. Congenital diaphragmatic hernia is a feature, albeit an uncommon one, of 22q11.2 deletion syndrome.

Taking the first point, first: it is worth considering that these normal prenatal microarray results, had they been available prior to delivery, might have discouraged the baby's physicians from ordering a chromosome microarray after delivery. In that scenario, a postnatal chromosome microarray might have been considered unnecessary at best and even redundant and wasteful. In retrospect, it is a good thing that the prenatal microarray results and the postnatal blood sample passed like ships in the night; otherwise, this copy number variant might not have been detected. When laboratories 


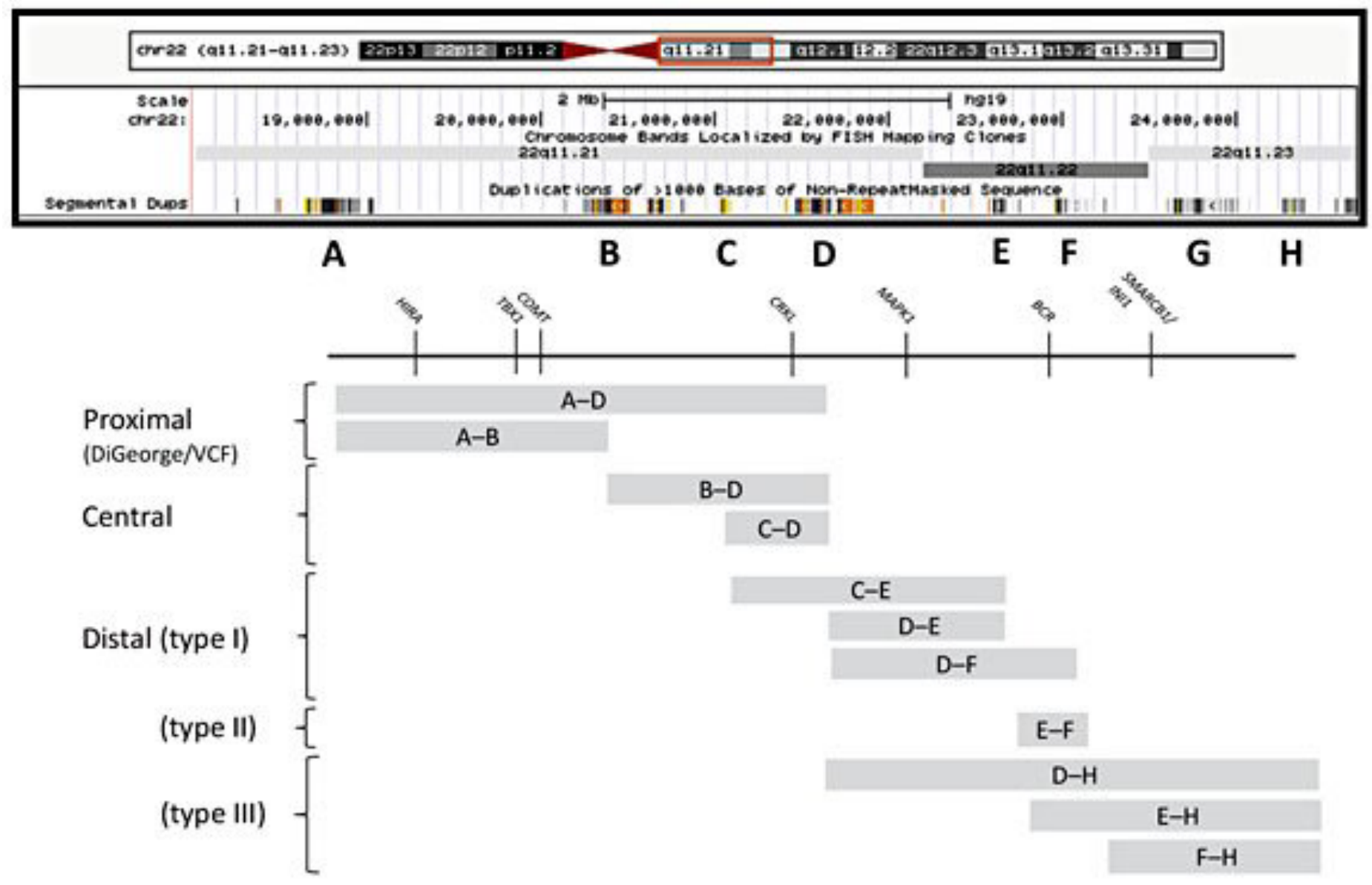

Figure 1: The proximal, central and distal 22q11.2 deletions are defined by their low copy repeat breakpoints. LCR22A-H and key genes are noted. (Adapted from Burnside, 2015)

have different reporting standards for prenatal and postnatal microarrays, and many do, a normal prenatal microarray result must carry less diagnostic weight. At the reference laboratory that processed this sample, and at others, deletions smaller than $1 \mathrm{Mb}$ are not reported on prenatal samples. This instance is not the first time that a postnatal microarray provided a diagnosis when the prenatal microarray was normal. After several such experiences, I no longer regard a normal prenatal microarray as a definitive test. Now I do not hesitate to order a postnatal microarray after a normal prenatal microarray when I suspect a chromosome anomaly.

Second, this child has one of the less commonly described $22 q 11.2$ deletion types, which are varied and numerous. 22q11.2 deletion syndrome (22q11.2DS) is the most common recurrent chromosome microdeletion syndrome with a prevalence of $1 / 2000$ $1 / 4000$ live births. The chromosome region around $22 q 11.2$ is predisposed to genomic instability because it is enriched with repetitive DNA sequences, called low copy repeats (LCR). A cluster of 8 LCRs, known as LCR22A-H, is responsible for chromosomal misalignment during meiotic recombination (nonallelic homologous recombination), which predisposes this region to recurrent deletions and duplications.

About $90 \%$ of patients with $22 q 11.2 \mathrm{DS}$ have a classic $\sim 3 \mathrm{Mb}$ deletion flanked by breakpoints in the two larger regions of repetition, LCR22A and LCR22D. Individuals with this 22q11.2 deletion commonly present with a recognizable facial phenotype, conotruncal heart defects, cleft palate, and features of DiGeorge syndrome or velocardiofacial syndrome.

The more distal $22 q 11.2$ deletions that lie farther from the centromere, with breakpoints from LCR22D to LCR22-H, are less common and have different phenotypes. Although these three distal 22q11.2 deletions have been lumped together (OMIM 611867), each probably represents a distinct clinical entity. They have been classified into three types: type I (LCR22C-F), type II (LCR22E-

The only worldwide monthly publication
exclusively serving Pediatric and Adult
Cardiologists that focus on Congenital/
Structural Heart Disease (CHD), and
CARGENITAL
TODAY $\quad \begin{aligned} & \text { Cardiothoracic Surgeons. } \\ & \text { Free on the Home Page }\end{aligned}$


F), and type III (deletions that include SMARCB1, LCR22D-H). Our patient has a type II distal deletion, which is the smallest and possibly mildest of the $22 q 11.2$ deletions. It does not share breakpoints with the classic LCR22A-D deletion and does not have many overlapping features other than intellectual disability.

The type II distal deletion is rare, with few reports in the literature. Mikhail et al. (2014) described 13 unrelated patients with various distal 22q11.2 deletions, who were ascertained by intellectual handicap, autism, dysmorphic features, or congenital anomalies. Of these, four patients had the smallest, type II, LCR22E-F deletion, similar to our patient. These individuals had a milder phenotype that did not include growth deficiency or cardiac defects. Burnside (2015) reviewed eight patients with type II distal deletions, including the four previously reported by Mikhail et al. in 2014 . Seven of the eight individuals (88\%) had developmental delay and dysmorphic features (abnormal ears, prominent forehead, or deep-set eyes). She described intellectual disability (4/8), CNS anomalies/seizures (2/8), hypotonia (1/8), and cardiovascular defect $(1 / 8)$.

As for the last point, $\mathrm{CDH}$ is a less common but recognized feature of 22q11.2DS. In their report of 28 fetuses with 22q11.2DS, Volpe et al. (2003) described 2/28 with diaphragmatic hernia. In their cohort of 1246 patients with 22q11.2DS from Children's Hospital of Philadelphia, Unolt et al. (2017) reported a prevalence of CDH of $0.8 \%(10 / 1246)$. All 10 of these children had other major malformations. All of the five in whom the breakpoints could be defined had the classic larger deletion flanked by LCR22 A-D. However, not all individuals with $\mathrm{CDH}$ have the classic $22 \mathrm{q} 11.2$ deletion. Stark et al. (2015) identified two different atypical 22q11.2 deletions that did not include $T B X 1$ among 28 individuals with $\mathrm{CDH}$ : one deletion was proximal to LCR22-B, and one was bordered by LCR22 C-D. Tan et al. (2011) reported CDH in a patient with a type I (LCR22D-E) distal 22q11.2 deletion. Although a candidate gene or critical region for diaphragmatic hernia has not been identified in the 22q11.2 region, our case may help narrow the region of interest.

\section{"Although a candidate gene or critical region for diaphragmatic hernia has not been identified in the 22q11.2 region, our case may help narrow the region of interest."}

\section{Practical Applications:}

1. Appreciate that 22q11.2 copy number variants (deletions and duplications) can be flanked by any two of the eight low copy repeats (LCR22A-H) that predispose this region to genomic instability.

2. Recognize that the most common (90\%) of the $22 q 11.2$ deletions is the classic $3 \mathrm{Mb} 22 q 11.2$ deletion (LCR22A-D) that presents with typical facial features, cardiac and palatal defects. However, expect about $10 \%$ of $22 q 11.2$ deletions to be atypical.

3. Appreciate that the three distal $22 q 11.2$ deletions are distinct entities with phenotypes that differ from the proximal classic deletion (LCR22A-D) associated with DiGeorge syndrome.

4. Be aware that prenatal and postnatal microarrays have different reporting standards, and small deletions or duplications may be missed prenatally.

5. Order a postnatal chromosome microarray when you suspect a copy number variant, regardless of normal prenatal microarray results.

6. Consider the $22 q 11.2$ deletion syndromes in the differential diagnosis of infants with a congenital diaphragmatic hernia, especially when there are other anomalies and/or dysmorphic features.

\section{References:}

1. Burnside RD. 22q11.21 Deletion Syndromes: A Review of Proximal, Central, and Distal Deletions and Their Associated Features. Cytogenet Genome Res. 2015;146(2):89-99. PMID: 26278718.

2. Mikhail FM, Burnside RD, Rush B, et al. The recurrent distal $22 q 11.2$ microdeletions are often de novo and do not represent a single clinical entity: a proposed categorization system. Genet Med. 2014 Jan;16(1):92-100. PMID: 23765049.

3. Stark Z, Behrsin J, Burgess T, et al. SNP microarray abnormalities in a cohort of 28 infants with congenital diaphragmatic hernia. Am J Med Genet A. 2015 Oct;167A(10):2319-26. PMID: 26059276

4. Tan TY, Collins A, James PA, et al. Phenotypic variability of distal 22q11.2 copy number abnormalities. Am J Med Genet A. 2011 Jul;155A(7):1623-33. PMID: 21671380

5. Unolt M, DiCairano L, Schlechtweg K, et al. Congenital diaphragmatic hernia in 22q11.2 deletion syndrome. Am J Med Genet A. 2017 Jan;173(1):135-142. PMID: 27682988

6. Volpe $P$, Marasini M, Caruso G, et al. $22 q 11$ deletions in fetuses with malformations of the outflow tracts or interruption of the aortic arch: impact of additional ultrasound signs. Prenat Diagn. 2003 Sep;23(9):752-7. PMID: 12975788.

Disclosures: The authors have no relevant disclosures.

\section{NT}

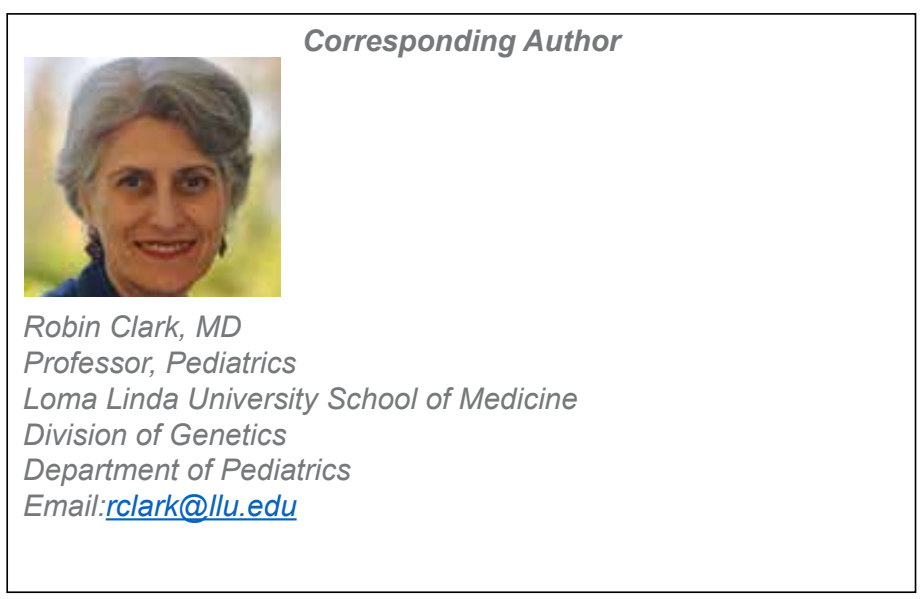

